\title{
Correction: Glioma-associated human endothelial cell-derived extracellular vesicles specifically promote the tumourigenicity of glioma stem cells via CD9
}

\author{
Dengke $\mathrm{Li}^{1} \cdot$ Yuan Tian ${ }^{1} \cdot$ Yan $\mathrm{Hu}^{1} \cdot$ Yingjiao $\mathrm{Qi}^{1} \cdot \mathrm{Ningyu} \operatorname{Tian}^{1} \cdot$ Shanshan $\mathrm{Li}^{1} \cdot$ Peishan $\mathrm{Hu}^{1} \cdot$ Fan $\mathrm{Wu}^{2}$. \\ Qunfang Wei ${ }^{1} \cdot$ Zhizhong Wei $^{1,3} \cdot$ Shanshan Wang ${ }^{1} \cdot$ Bin Yin ${ }^{1} \cdot$ Tao Jiang ${ }^{2,4} \cdot$ Jiangang Yuan $^{1} \cdot$ Boqin Qiang $^{1}$. \\ Wei Han ${ }^{1} \cdot$ Xiaozhong Peng ${ }^{1,3}$
}

Published online: 19 September 2019

(c) The Author(s), under exclusive licence to Springer Nature Limited 2019

\section{Correction to: Oncogene}

https://doi.org/10.1038/s41388-019-0903-6

The original version of this Article contained errors in the author affiliations.

The affiliations for Xiaozhong Peng should be State Key Laboratory of Medical Molecular Biology and Department of Molecular Biology and Biochemistry, Institute of Basic
Medical Sciences, Medical Primate Research Center, Neuroscience Center, Chinese Academy of Medical Sciences, School of Basic Medicine Peking Union Medical College, Beijing, China and Institute of Medical Biology, Chinese Academy of Medical Sciences, Peking Union Medical College, Kunming, China.

This error has been corrected in both the PDF and HTML versions of the Article.
The original article can be found online at https://doi.org/10.1038/ s41388-019-0903-6.

Wei Han

hanwei2012@ibms.pumc.edu.cn

$\triangle$ Xiaozhong Peng

pengxiaozhong@pumc.edu.cn

peng_xiaozhong@163.com

1 State Key Laboratory of Medical Molecular Biology, Department of Molecular Biology and Biochemistry, Institute of Basic Medical Sciences, Medical Primate Research Center, Neuroscience Center, Chinese Academy of Medical Sciences, School of Basic Medicine Peking Union Medical College, Beijing, China

2 Department of Molecular Neuropathology, Beijing Neurosurgical Institute, Capital Medical University, Beijing, China

3 Institute of Medical Biology, Chinese Academy of Medical Sciences, Peking Union Medical College, Kunming, China

4 Department of Neurosurgery, Beijing Tiantan Hospital, Capital Medical University, Beijing, China 